\title{
The role of SDF-I/CXCR4 in the vasculogenesis and remodeling of cerebral arteriovenous malformation
}

\author{
This article was published in the following Dove Press journal: \\ Therapeutics and Clinical Risk Management \\ I September 2015 \\ Number of times this article has been viewed
}

\author{
Lingyan Wang' \\ Shaolei Guo ${ }^{2}$ \\ Nu Zhang ${ }^{2}$ \\ Yuqian $\mathrm{TaO}^{3}$ \\ Heng Zhang' \\ Tiewei $\mathrm{Qi}^{2}$ \\ Feng Liang ${ }^{2}$ \\ Zhengsong Huang ${ }^{2}$ \\ 'Department of Neurosurgery \\ ICU, ${ }^{2}$ Department of Neurosurgery, \\ ${ }^{3}$ Department of Neurology, The First \\ Affiliated Hospital of Sun Yat-Sen \\ University, Guangzhou, People's \\ Republic of China
}

Correspondence: Zhengsong Huang Department of Neurosurgery, The First Affiliated Hospital of Sun Yat-Sen University, Number 58 Zhongshan Second Road, Guangzhou 510080 ,

People's Republic of China

Tel +86202882 3388

Fax +86 2028823388

Email zs_huang@yeah.net
Background: Cerebral arteriovenous malformation (AVM) involves the vasculogenesis of cerebral blood vessels and can cause severe intracranial hemorrhage. Stromal cell-derived factor-1 (SDF-1) and its receptor, CXCR4, are believed to exert multiple physiological functions including angiogenesis. Thus, we investigated the role of SDF-1/CXCR4 in the vasculogenesis of cerebral AVM.

Methods: Brain AVM lesions from surgical resections were analyzed for the expression of SDF-1, CXCR4, VEGF-A, and HIF-1 by using immunohistochemical staining. Flow cytometry was used to quantify the level of circulating endothelial progenitor cells (EPCs). Further, in an animal study, chronic cerebral hypoperfusion model rats were analyzed for the expression of SDF-1 and HIF-1. CXCR4 antagonist, AMD3100, was also used to detect its effects on cerebral vasculogenesis and SDF-1 expression.

Results: Large amounts of CXCR4-positive CD45 cells were found in brain AVM lesion blood vessel walls, which also have higher SDF-1 expression. Cerebral AVM patients also had higher level of EPCs and SDF-1. In chronic cerebral hypoperfusion rats, SDF-1, HIF-1, and CD45 expressions were elevated. The application of AMD3100 effectively suppressed angiogenesis and infiltration of CXCR4-positive $\mathrm{CD}^{4} 5^{+}$cells in hypoperfusion rats compared to controls.

Conclusion: The SDF-1/CXCR4 axis plays an important role in the vasculogenesis and migration of inflammatory cells in cerebral AVM lesions, possibly via the recruitment of bone marrow EPCs.

Keywords: cerebral arteriovenous malformation, SDF-1/CXCR4, chronic cerebral hypoperfusion, endothelial progenitor cells

\section{Introduction}

As the most commonly occurring cerebral vessel malformation, cerebral arteriovenous malformation (AVM) has an approximate incidence of $0.010 \%-0.018 \%$ in the whole population. ${ }^{1}$ It is astonishing that only $12 \%$ of AVM present symptoms during life; ${ }^{2}$ thus, the actual occurrence frequency of brain AVM may be ten times more than the epidemiological data. About half the cerebral AVM patients have their disease onset as intracranial hemorrhage, representing about $1.4 \%-2 \%$ of the total hemorrhagic brain stroke. ${ }^{3}$ Most brain AVM-related hemorrhages occur before patients reach their 50s, ${ }^{4}$ making it a major reason for mortality and disability in young adults. Therefore, effective prevention of brain AVM is of great importance and requires a comprehensive understanding of the pathogenesis, which, however, remains unclear at the current stage. More and more evidence has shown that, instead of a stable lesion, cerebral AVM forms and develops in a dynamic vessel remodeling and vasculogenesis 
process. ${ }^{5}$ Therefore, the knowledge of molecular mechanism underlying vasculogenesis and remodeling of brain AVM may help to develop novel therapeutic approaches.

Stromal cell-derived factor-1 (SDF-1) belongs to the endocrine type of CXC chemokines ${ }^{6}$ and has been reported to play important roles in various physiological functions, including inflammatory response, hematopoietic stem cell mobilization, metastasis of malignant tumors, and vasculogenesis, through interaction with its specific receptor CXCR4 ${ }^{7,8}$ The detailed mechanism of SDF-1/CXCR4 in facilitating vasculogenesis is mainly via its synergistic effects with vascular endothelial growth factor (VEGF) to stimulate the proliferation and tube formation of endothelial cells by mobilizing, recruiting, and homing CXCR4 bone marrow-derived cells including 1) endothelial progenitor cells (EPCs), which could differentiate into mature endothelial cells; 2) pericyte progenitor cells; 3) smooth muscle progenitor cells; and 4) bone marrow-derived $\mathrm{CD} 45^{+}$vascular modulatory cells, which act as vascular modulators by differentiating into mature endothelial cells and smooth muscle cells, and by secreting proangiogenesis factors. Meanwhile, SDF-1 could induce proliferation and differentiation of endothelial cells, thus regulating the secretion of angiogenesis-related cytokines and exerting a synergistic effect with VEGF on inducing neo-angiogenesis. ${ }^{9}$ Therefore, SDF-1 played an important role in angiogenesis and vessel remodeling in both embryonic and postnatal development.

This study aimed at investigating the relationship between SDF-1 /CXCR4 and pathogenesis of AVM. Clinical samples of AVM lesions were studied for the expression profile of SDF- $1 \alpha$ and CXCR4, in addition to the quantification of bone marrow-derived EPCs in patients. Such a human model was also replicated in AVM-related chronic cerebral hypoperfusion ( $\mathrm{CCH})$ animals. In this study, we investigated the expression of SDF-1 in cerebral AVM lesions by immunohistochemistry, and explored whether EPCs and hematopoieticderived inflammatory cells $\left(\mathrm{CD} 45^{+}\right.$cells) were present in AVM lesions by immunofluorescence. Furthermore, we evaluated the effect of SDF-1/CXCR4 on neo-angiogenesis in rat cerebral chronic hypoperfusion models.

\section{Materials and methods Expression of SDF-I $\alpha$ and CXCR4 in human cerebral AVM lesions}

A total of 48 cerebral AVM samples were collected from surgical resections in the Department of Neurosurgery, The First Affiliated Hospital of Sun Yat-Sen University, Guangzhou, People's Republic of China, between January 2012 and
December 2013. Among all 48 patients, there were 33 males and 15 females, with an average age $=30.66 \pm 11.90$ years. The location of AVM included the cerebellum (five cases) and the supratentorial structures (43 cases). Another five brain tissue samples with normal blood formation were collected from patients undergoing trauma surgery and temporal lobectomy for seizures. General condition of patients, in addition to hemorrhagic volume, Spetzer-Martin grade, and preoperative embolotherapy, were recorded. All procedures have been approved by the ethical committee of The First Affiliated Hospital of Sun Yat-Sen University. Written consents were obtained from patients or families.

Collected brain tissues were embedded in paraffin and sectioned into $10 \mu \mathrm{m}$ thickness tissue slices, which were mounted on glass slides. Immunohistochemical (IHC) staining method was adopted as previously reported. ${ }^{10}$ For the single labeling, we identified the expression of SDF- $1 \alpha$, CXCR4, VEGF-A, and HIF-1 $\alpha$ by using rabbit antihuman antibody (Santa Cruz Biotechnology, Dallas, TX, USA) and biotinylated goat antirabbit immunoglobin $\mathrm{G}$ ( $\operatorname{IgG}$ ) (Abcam, Cambridge, MA, USA), and ABC streptavidin detection kit (Vector Labs, Burlingame, CA, USA). For the double-fluorescent staining, Alexa Fluor 594- or 488-labeling secondary antibody (Invitrogen, Waltham, MA, USA) was adopted after primary antibody (CXCR4 plus CD45, or CD133 plus CD34) incubation to identify the origin of those positive cells.

\section{Identification of peripheral EPCs and SDF-I $\alpha$ expression}

A total of 15 AVM patients were randomly selected for venous blood collection, along with 15 healthy controls. Flow cytometry technique was applied to analyze the percentage of EPCs in the total mononuclear cells as previously reported. ${ }^{11}$ EPCs were defined as triple-positive signals for CD133, CD34, and KDR. Enzyme-linked immunosorbent assay (ELISA) was also employed to detect the level of SDF-1 $\alpha$ in peripheral blood by using previously established protocols $^{12}$ and the human CXCL12/SDF-1 $\alpha$ ELISA kit (R\&D Systems, Minneapolis, MN, USA). A correlation analysis was then performed to identify the correlation between EPCs percentage and SDF-1 $\alpha$ level in each individual sample.

\section{Expression of SDF-I $\alpha$ and vasculogenesis in chronic cerebral hypoperfusion rat models}

A cohort of 54 male Sprague Dawley (SD) rats (average age $=8$ weeks old, body weight between 200 and $250 \mathrm{~g}$ ) were divided into model, sham, and control group ( $\mathrm{n}=18$ each). 
The $\mathrm{CCH}$ model was prepared as described by Hai et al. ${ }^{13}$ In brief, after anesthesia, an end-to-side anastomosis between the right distal external jugular vein and the ipsilateral common carotid artery was made, followed by the ligation of the left vein that drains the transverse sinus and the bilateral external carotid arteries. After recovery, ${ }^{18} \mathrm{~F}$-labeled 2-dexoxy-2-fluoro-D-glucose (FDG) PET/CT (positron emission tomography/computed tomography) scan was used to evaluate the metabolic activity of rat brains as shown by the intake level of ${ }^{18} \mathrm{~F}$-FDG. All animal experimental procedures were approved by the animal research committee of the host institute.

\section{Effects of AMD3 100 in blocking SDF-I/CXCR4 pathway and its role in vasculogenesis}

Another 16 male SD rats (age and weight comparable to those in the previous experiment) were prepared by using the same CCH methods. After recovery, AMD3100 (5 mg/kg/d) or equal volume of saline was injected by the intraperitoneal route daily for 30 consecutive days. Another eight rats with comparable age and body weights were employed as the control group. At the end of drug treatment, all rats were sacrificed and their brains were collected. IHC staining using CD34 antibody was applied to determine microvessel density (MVD), followed by CD45/CXCR4 double fluorescent IHC staining and Western blot for MMP-9 proteins.

\section{Statistical analysis}

All collected data were analyzed by SPSS version 17 software package and presented as mean \pm SEM. Between-group comparisons were performed by two-sample Student's $t$-test, while multiple group comparisons were achieved using analysis of variance. A statistical significance is defined as $P<0.05$.

\section{Results \\ SDF- I/CXCR4 was abundantly expressed in cerebral AVM lesions}

We found a higher SDF-1 $\alpha$ protein expression in all 48 AVM patients, and 40 cases (83.3\%) had CXCR4-positive staining (Figure 1A and B). Further examination showed that SDF-1 $\alpha$ was mainly expressed in artery smooth muscles and vessel endothelial cells, while CXCR4 was present in the inner wall of AVM-deformed vessels and endothelial cells. Double-fluorescent IHC images showed the colocalization of CD45/CXCR4 in malformed vascular walls in peripheral matrix (Figure 1C). We also identified the positive correlation between SDF- $1 \alpha$ and VEGF-A or HIF-1 $\alpha$, with correlation coefficient $r$ at 0.537 and 0.437 , respectively $(P<0.05$ in both cases). A further consideration of patients' clinical data found that the intensity of SDF-1 $\alpha$ expression was stronger in patients with preoperative embolotherapy $(P<0.05)$.

\section{Higher numbers of EPCs and SDF-I $\alpha$ expression in AVM lesions}

The flow cytometry results showed that in AVM patients, the double-positive cells (CD133 and CD34) and triplepositive cells (CD133, CD34, and KDR) accounted for $0.231 \% \pm 0.179 \%$ and $0.051 \% \pm 0.029 \%$ of total mononuclear cells in peripheral blood, significantly higher than $0.093 \% \pm 0.045 \%$ and $0.023 \% \pm 0.016 \%$ in healthy controls (Figure 2, $P<0.05$ in both comparisons). The level of SDF- $1 \alpha$ was also elevated from $1,432 \pm 331.7 \mathrm{pg} / \mathrm{mL}$ in healthy control to $2,003.3 \pm 540.6 \mathrm{pg} / \mathrm{mL}$ in AVM patients $(P<0.05)$. The number of EPCs and serum SDF-1 $\alpha$ level showed a strong positive relationship $(r=0.79, P<0.05)$. We also found double-positive EPCs (CD133/CD34) in AVM lesions (Figure 1D).

\section{SDF-I $\alpha$ expression was elevated in chronic cerebral hypoperfusion rats}

The MVD in the cerebral middle artery-feeding cortex was significantly higher in model rats at 7, 42, or 90 days when compared to that in sham group (Table $1, P<0.05$ in all cases). The images of ${ }^{18} \mathrm{~F}-\mathrm{FDG}$ PET/CT scan showed a dynamic change in cerebral intake of ${ }^{18} \mathrm{~F}$-FDG in rats model as the SUV ${ }_{\text {max }}$ value significantly increased 24 hours after the surgery, and decreased at day 7 , followed by increase at day 42 and day 90. Those values, however, were still lower than those of control or sham group (Table 2, $P<0.05$ ). The serum level of SDF-1 $\alpha$ started to increase 24 hours after the surgery, reached a peak at day 7 , and was still higher than control group at day 42 and day 90 (Table $3, P<0.05$ ). A further IHC examination showed the presence of large amounts of CD45 ${ }^{+}$ cells in cerebral tissues in model rats (Figure 3).

\section{SDF-I/CXCR4 pathway inhibitor AMD3 100 effectively inhibited vasculogenesis in AVM rats}

The MVD values in control, AMD3100-, and saline-treated group after a 30-day treatment were $63.53 \pm 9.89 / \mathrm{mm}^{2}$, $82.50 \pm 12.31 / \mathrm{mm}^{2}$, and $129.70 \pm 19.72 / \mathrm{mm}^{2}$, respectively $(P<0.05)$. The average number of double-positive cells (CD45 and CXCR4) in each high magnification field were 
A

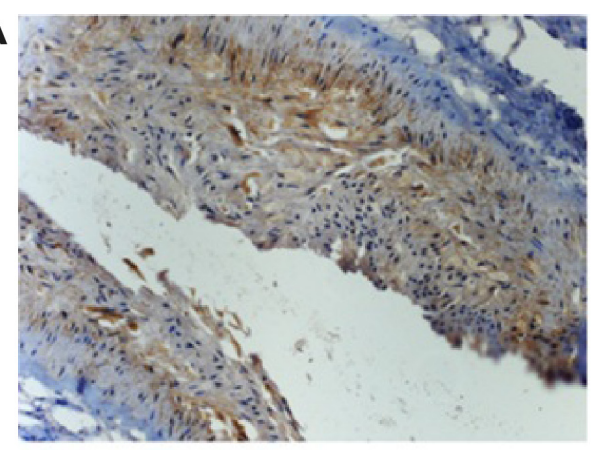

B
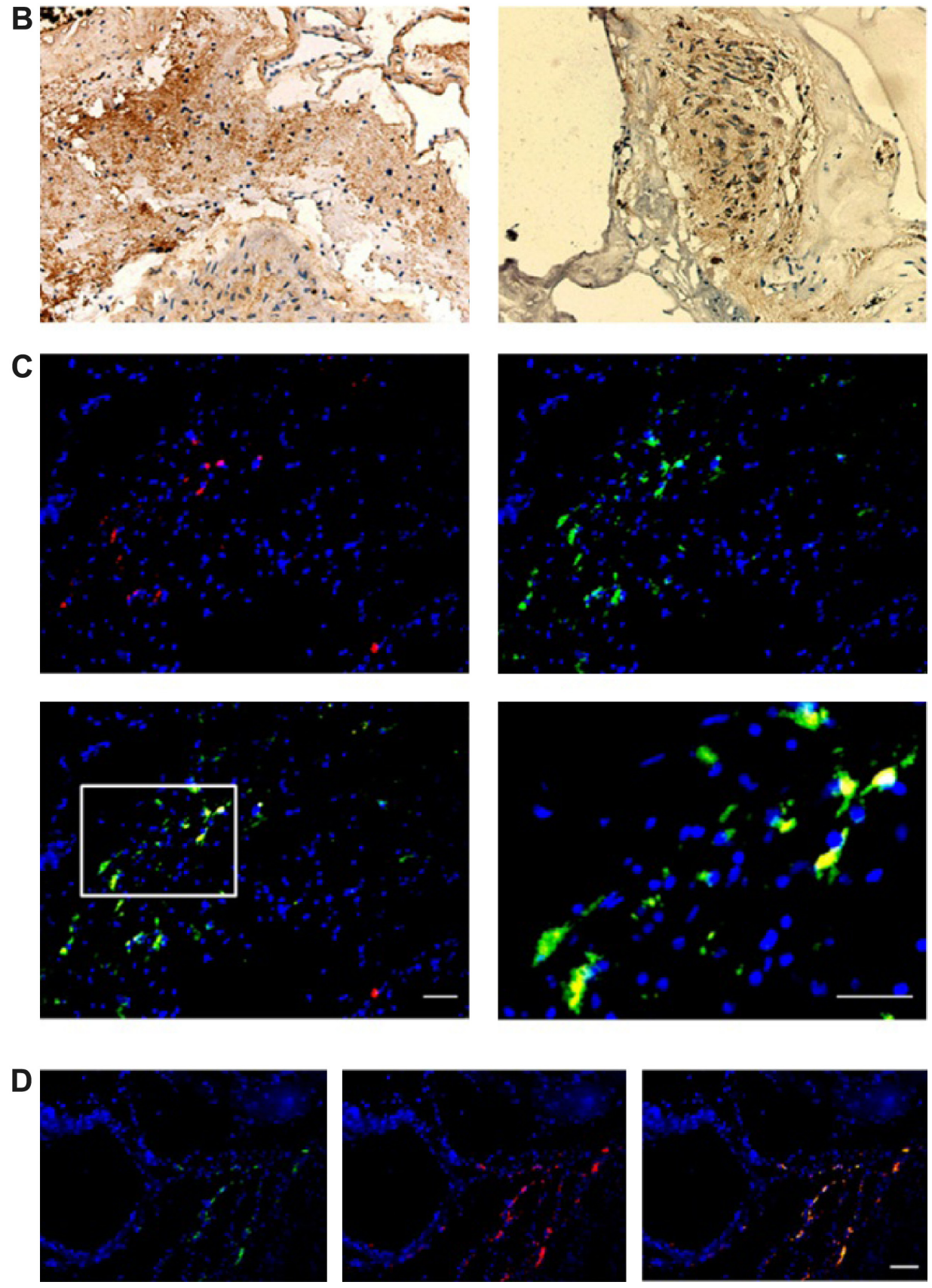

Figure I SDF-I $\alpha$ and CXCR4 expression in AVM lesions.

Notes: (A) IHC staining of SDF-I $\alpha, \times 200$. (B) CXCR4 IHC staining image, $\times 200$. (C) Double immunofluorescence images of CXCR4 (red) and CD45 (green). Scale bar $=20 \mu \mathrm{m}$. The white box is magnified in the right panel. (D) Double staining of CDI33 (left, green) and CD34 (middle, red). Scale bar =50 $\mu \mathrm{m}$. Abbreviations: IHC, immunohistochemistry; AVM, arteriovenous malformation; SDF-I, stromal cell-derived factor-I. 

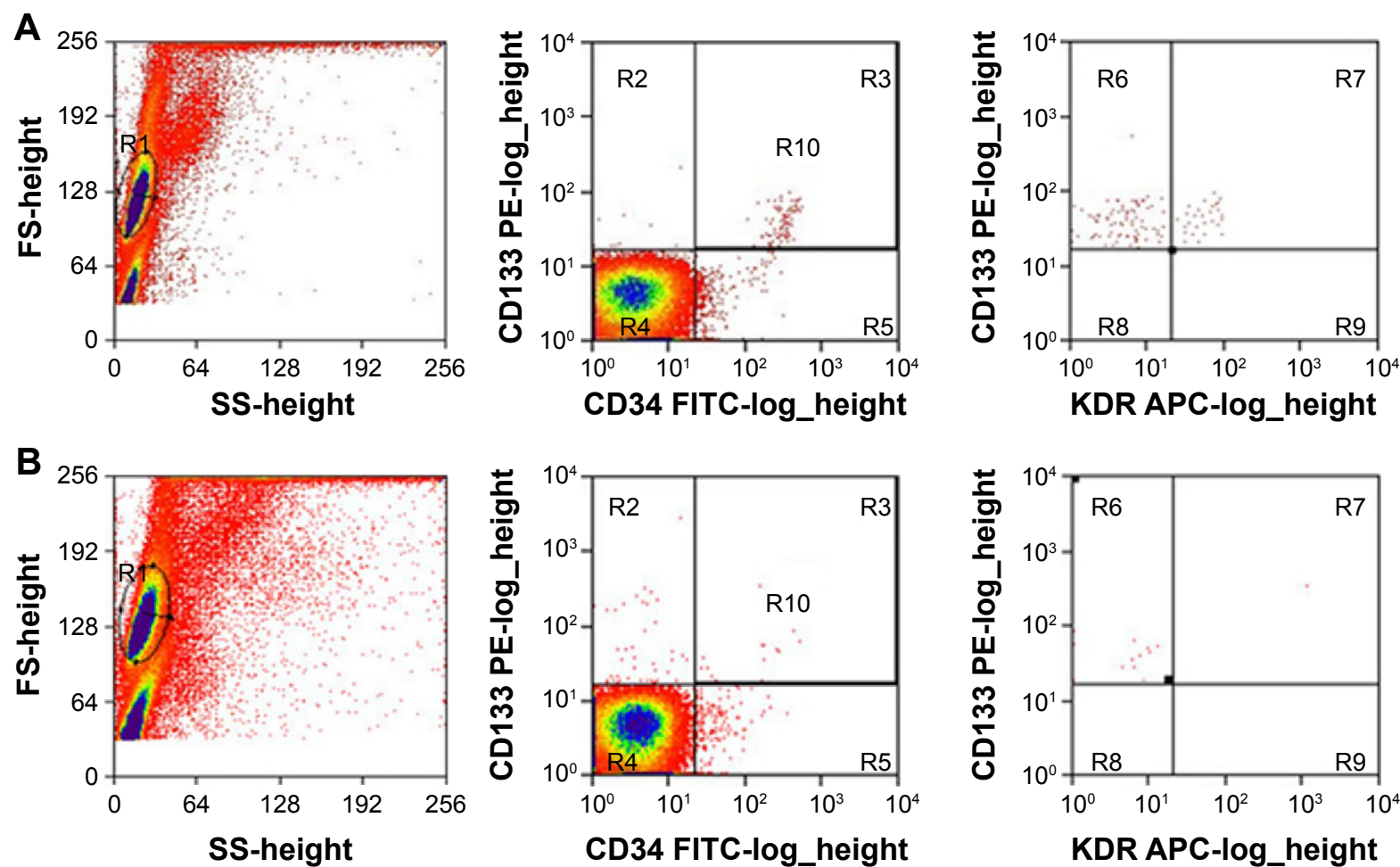

Figure 2 EPCs from peripheral blood.

Notes: This figure showed flow cytometry results of AVM patients (A); in controls (B). Left panels, raw images; middle panels, CDI33+/CD34+ double-positive cells (upper

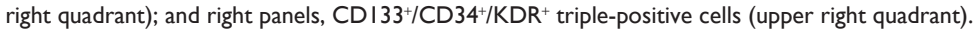

Abbreviations: EPCs, endothelial progenitor cells; AVM, arteriovenous malformation.

$3.83 \pm 1.47,7.33 \pm 2.16$, and 11.33 \pm 3.61 in control, AMD3100-, and saline-treated group, respectively $(P<0.05)$ (Figure 4). The MMP-9 protein expression also showed the same pattern, which was higher than in control ones but lower than in saline-treated animals (Figure 5, $P<0.05$ ).

\section{Discussion}

This study conducted both clinical and animal researches to establish the role of SDF-1/CXCR4 pathway in the vasculogenesis of AVM, possibly through the mobilization of bone marrow-derived EPCs. In the clinical study, we found a strong expression of CXCR4 in $\mathrm{CD} 45^{+}$cells, which are possibly inflammatory cells, by chemotrophic attractions by SDF-1 $\alpha$, such as mononuclear cells from bone marrows. This can be supported by the following two experiments: 1) higher number of EPCs and higher quantity of serum

Table I Microvessel density $\left(/ \mathrm{mm}^{2}\right)$ between sham and model animals

\begin{tabular}{lllll}
\hline & Sham & Model & $\boldsymbol{t}$ & P-value \\
\hline $24 \mathrm{~h}$ & $56.17 \pm 8.06$ & $65.64 \pm 8.57$ & 1.974 & 0.077 \\
$7 \mathrm{~d}$ & $55.13 \pm 12.59$ & $92.67 \pm 11.12$ & 5.472 & 0.000 \\
$42 \mathrm{~d}$ & $59.92 \pm 7.55$ & $144.66 \pm 14.87$ & 12.488 & 0.000 \\
$90 \mathrm{~d}$ & $54.88 \pm 11.93$ & $143.78 \pm 15.33$ & 11.207 & 0.000 \\
\hline
\end{tabular}

Note: Data are represented as mean \pm standard deviation. Abbreviations: h, hours; d, days.
SDF-1 $\alpha$ occurred in AVM patients; and 2) SDF-1 $\alpha$ expression was also elevated in rats after $\mathrm{CCH}$. The blockage of SDF-1/CXCR4 pathway by using AMD3100 effectively inhibited either vasculogenesis or CD45/CXCR doublepositive cell numbers in the brain cortex. All these results propose a model in which SDF-1 $\alpha$, after its induction by hypoperfusion, binds to CXCR4 and recruit EPCs for vasculogenesis in AVM lesions.

SDF-1 and its specific receptor, CXCR4, are expressed in various tissues, including hematopoietic and endothelial cells. ${ }^{8}$ Studies have demonstrated the critical role of SDF-1/ CXCR4 pathway in the trafficking and engraftment of hematopoietic stem cells from bone marrow into the general circulation. ${ }^{14}$ The role of this pathway in embryonic

Table 2 SUV $_{\max }$ value of left MCA cortex in control group and model groups

\begin{tabular}{|c|c|c|c|c|c|}
\hline \multirow[t]{2}{*}{ Normal } & \multicolumn{4}{|c|}{ Model } & \multirow[t]{2}{*}{ P-value } \\
\hline & $24 \mathrm{~h}$ & $7 d$ & $42 \mathrm{~d}$ & $90 \mathrm{~d}$ & \\
\hline \multicolumn{6}{|c|}{ 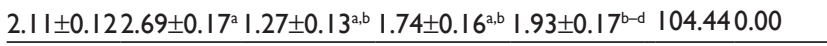 } \\
\hline \multicolumn{6}{|c|}{$\begin{array}{l}\text { Notes: Data are represented as mean } \pm \text { standard deviation. }{ }^{a} P<0.05 \text { compared to } \\
\text { control; }{ }^{p} P<0.05 \text { compared to those at } 24 \mathrm{~h} ;{ }^{c} P<0.05 \text { compared to those at } 7 \mathrm{~d} \text {; } \\
{ }^{d} \mathrm{P}<0.05 \text { compared to those at } 42 \mathrm{~d} \text {. }\end{array}$} \\
\hline \multicolumn{6}{|c|}{$\begin{array}{l}\text { Abbreviations: } h \text {, hours; } d \text {, days; } S V_{\text {max }} \text {, maximum standardized uptake value; } \\
\text { MCA, middle cerebral artery. }\end{array}$} \\
\hline
\end{tabular}


Table 3 Serum SDF-I $\alpha$ levels $(\mathrm{pg} / \mathrm{mL})$

\begin{tabular}{lllll}
\hline & Sham & Model & $\boldsymbol{t}$ & $\boldsymbol{P}$ \\
\hline $24 \mathrm{~h}$ & $397.7 \pm 27.9$ & $450.3 \pm 40.4$ & 2.624 & 0.025 \\
$7 \mathrm{~d}$ & $406.2 \pm 32.3$ & $603.7 \pm 63.6$ & $6.78 \mathrm{I}$ & 0.000 \\
$42 \mathrm{~d}$ & $406.5 \pm 40.0$ & $564.8 \pm 36.4$ & 7.259 & 0.000 \\
$90 \mathrm{~d}$ & $387.0 \pm 51.9$ & $481.8 \pm 35.5$ & 3.699 & 0.004 \\
\hline
\end{tabular}

Note: Data are represented as mean \pm standard deviation.

Abbreviations: SDF-I, stromal cell-derived factor-I; h, hours; d, days.

vasculogenesis has also been proven as SDF-1 and CXCR4 double-knockout mice had severe blood vessel abnormalities. ${ }^{15}$ This study showed abundant expression of SDF-1 and CXCR4 proteins in AVM lesions from both human samples and mouse models, suggesting the role of this chemokine and its receptor in neo-angiogenesis, just as those in various tumor tissues. ${ }^{16}$ Little has been known, however, of the mechanism of SDF-1 in exerting the angiogenic effects, nor did the effect of CXCR4 inhibitor, AMD3100, in hematopoietic stem cell mobilization or angiogenic functions.

This study partially resolved the aforementioned question by applying AMD3100 in $\mathrm{CCH}$ rats and found depressed vasculogenesis and CXCR4-positive cells, suggesting that under hypoxic conditions, SDF-1 can recruit bone marrow-derived cells for vasculogenesis. This result is consistent with previous studies, in which SDF-1 was found to be upregulated in damaged liver, ${ }^{17}$ artery, ${ }^{18}$ bone marrow, ${ }^{19}$ and ischemic tissues. ${ }^{20}$ The research conducted on ischemic tissues ${ }^{20}$ also indicated that the upstream regulator of SDF-1 under hypoxic conditions is HIF-1. In this study, we found a positive correlation between SDF- $1 \alpha$ and HIF- $1 \alpha$ in AVM-related vascular walls, for the first time suggesting the pathway of HIF-1/SDF-1/CXCR4 axis in the malformation and neo-angiogenesis in AVM lesions.

SDF-1 has been reported to be involved in the cell proliferation, differentiation, and tube formation of vascular endothelial cells in vitro. ${ }^{21}$ The in vivo study supported a synergistic effect between SDF-1 and VEGF-A in angiogenesis. ${ }^{22}$ This study also discovered a positive relationship between SDF-1 and VEGF-A in AVM lesions, suggesting their important roles in AVM-related vessel malformation. In an ischemic limb model, SDF-1/CXCR4 pathway requires VEGF-A for its activation for angiogenesis. ${ }^{23}$ Therefore, it is reasonable to believe that VEGF-A and SDF-1 exert a synergistic effect in AVM malformation of blood vessels.

A previous study has suggested that brain and spinal AVM tissues had higher levels of SDF-1 and EPCs. ${ }^{10}$ This study, however, did not answer the question about how SDF-1 exerts its role in vasculogenesis. This study largely agreed with them by showing potent SDF-1 expression in vascular walls of cerebral AVM lesions, and by further showing the MMP-9 and VEGF-A proteins postulated both upstream and downstream factors in this regulatory pathway. We also, for the first time, replicated such proposed model in $\mathrm{CCH}$ rats, thereby further strengthening the initial hypothesis via the usage of specific antagonist AMD3100.

In summary, in cerebral AVM, neo-angiogenesis may occur via the activation of SDF-1/CXCR4 pathway, which further recruit bone marrow-derived EPCs to accomplish the vascular formation. This study, however, did not reveal the detailed cellular mechanism underlying this process. Future experiments can include specific cell markers to
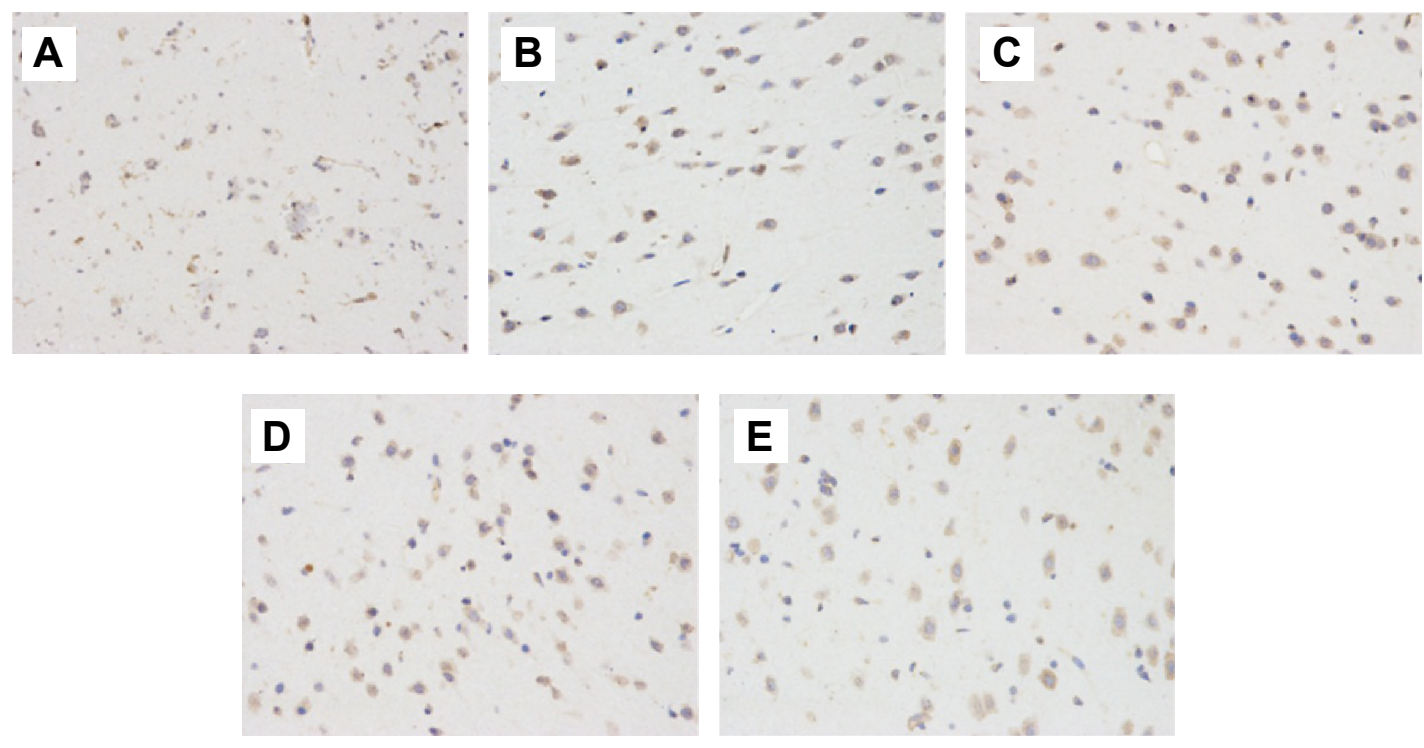

Figure $3 \mathrm{CD} 45^{+}$expression in chronic hypoperfusion mice.

Notes: (A) Control; (B) 24 hours after surgery; (C) 7 days; (D) 42 days; and (E) 90 days after surgery. Magnification: $\times 400$. 

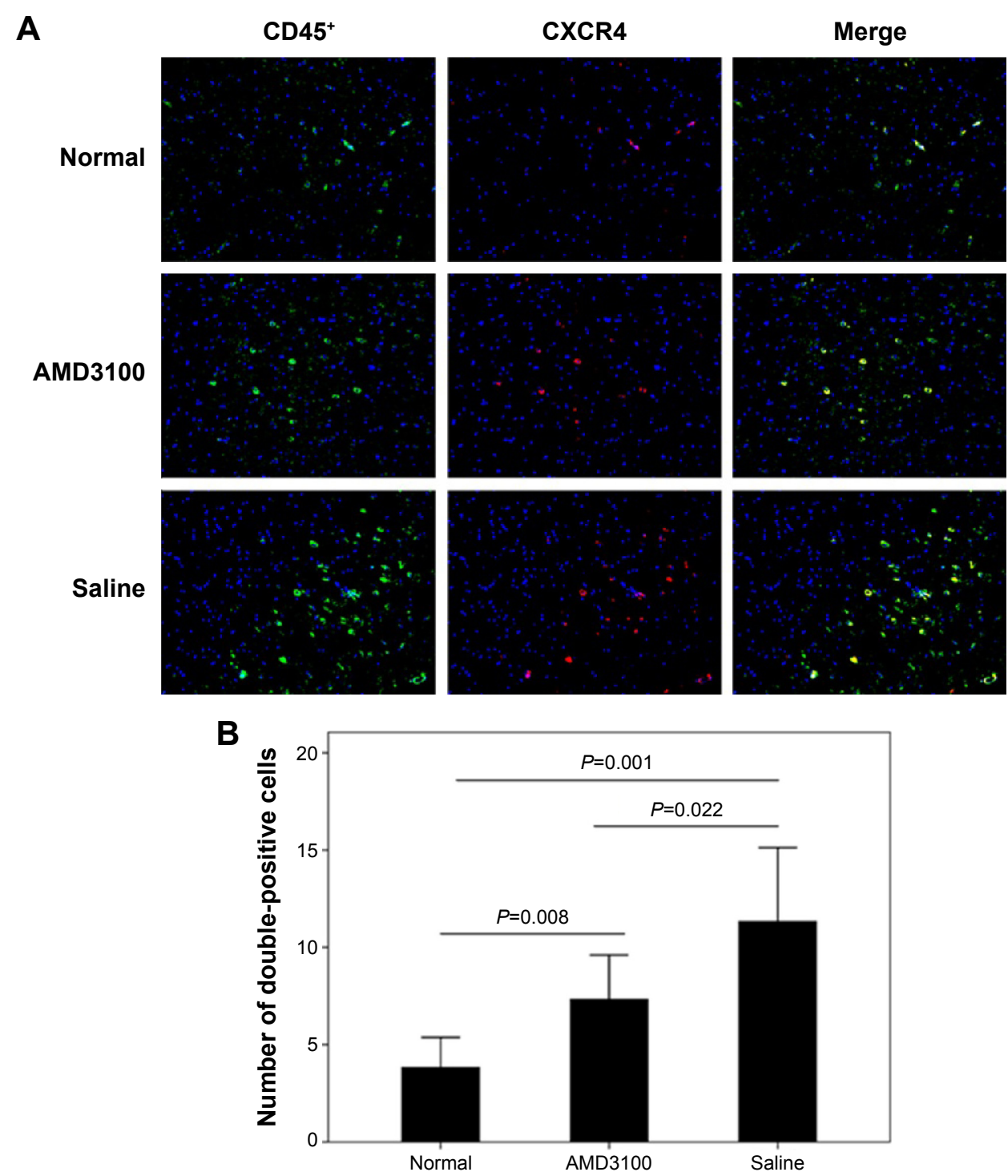

Figure 4 CD45/CXCR4 coexpression in AMD3100-treated mice.

Notes: (A) This figure shows representative double-immunofluorescence staining of CD45 (green) and CXCR4 (red) in AVM tissues. (B) This shows the quantified results of double-positive cells across control, AMD3100-, and saline-treated animals.

Abbreviation: AVM, arteriovenous malformation.

A

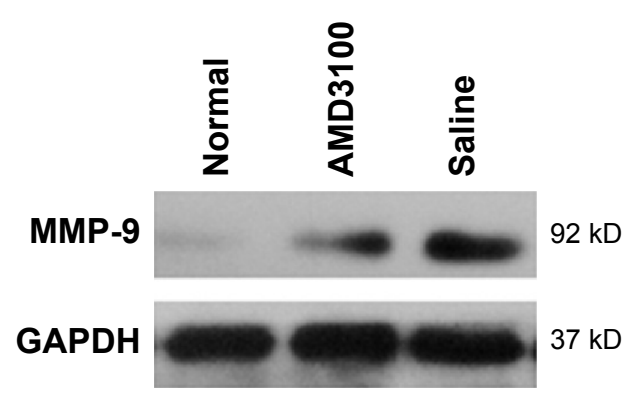

B

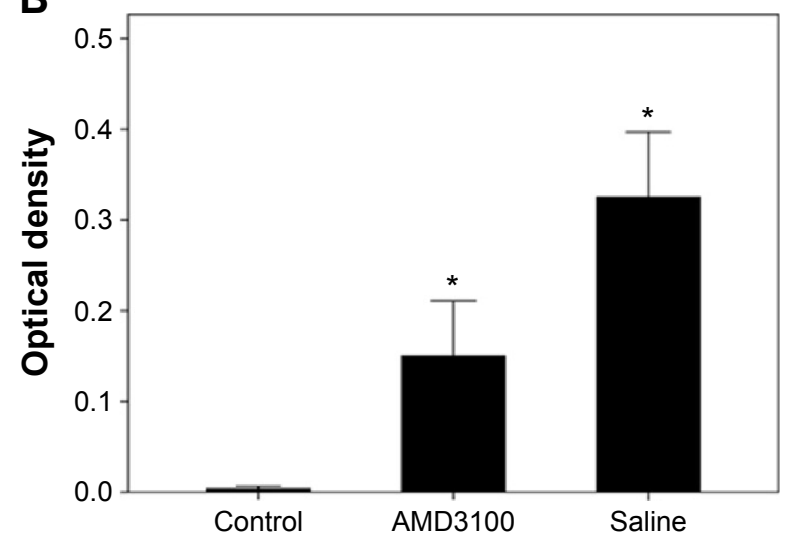

Figure 5 MMP-9 expression in AMD3100-treated mice.

Notes: (A) Western blotting bands. (B) Quantified results (in optical density) showing MMP-9 protein levels in control, AMD3I00-, and saline-treated animals. $* P<0.05$ compared to the normal ones. 
trace the migration of EPCs in the vascular formation under hypoxic conditions in animal models.

\section{Acknowledgment}

This work was supported by the National Natural Science Foundation of China (81171102).

\section{Disclosure}

The authors report no conflicts of interest in this work.

\section{References}

1. The Arteriovenous Malformations Study Group. Arteriovenous malformations of the brain in adults. N Engl J Med. 1999;340:1812-1818.

2. McCormick W. Classification, pathology, and natural history of angiomas of the central nervous system. Wkly Update Neurol Neurosurg. 1978; $14: 2-7$.

3. Stapf C, Labovitz DL, Sciacca RR, Mast H, Mohr JP, Sacco RL. Incidence of adult brain arteriovenous malformation hemorrhage in a prospective population-based stroke survey. Cerebrovasc Dis. 2001;13: 43-46.

4. Brown RD Jr, Wiebers DO, Torner JC, O'Fallon WM. Frequency of intracranial hemorrhage as a presenting symptom and subtype analysis: a population-based study of intracranial vascular malformations in Olmsted County, Minnesota. J Neurosurg. 1996;85:29-32.

5. Shenkar R, Elliott JP, Diener K, et al. Differential gene expression in human cerebrovascular malformations. Neurosurgery. 2003;52: 465-477; discussion 477-478.

6. Oberlin E, Amara A, Bachelerie F, et al. The CXC chemokine SDF-1 is the ligand for LESTR/fusin and prevents infection by T-cell-line-adapted HIV-1. Nature. 1996;382:833-835.

7. Nagasawa T, Tachibana $\mathrm{K}$, Kishimoto $\mathrm{T}$. A novel CXC chemokine PBSF/SDF-1 and its receptor CXCR4: their functions in development, hematopoiesis and HIV infection. Semin Immunol. 1998;10:179-185.

8. Petit I, Jin D, Rafii S. The SDF-1-CXCR4 signaling pathway: a molecular hub modulating neo-angiogenesis. Trends Immunol. 2007;28:299-307.

9. Tang JM, Wang JN, Zhang L, et al. VEGF/SDF-1 promotes cardiac stem cell mobilization and myocardial repair in the infarcted heart. Cardiovasc Res. 2011;91:402-411.
10. Gao P, Chen Y, Lawton MT, et al. Evidence of endothelial progenitor cells in the human brain and spinal cord arteriovenous malformations. Neurosurgery. 2010;67:1029-1035.

11. Rustemeyer P, Wittkowski W, Jurk K, Koller A. Optimized flow cytometric analysis of endothelial progenitor cells in peripheral blood. J Immunoassay Immunochem. 2006;27:77-88.

12. Di Marco GS, Rustemeyer P, Brand M, et al. Circulating endothelial progenitor cells in kidney transplant patients. PLoS One. 2011;6:e24046.

13. Hai J, Li ST, Lin Q, Pan QG, Gao F, Ding MX. Vascular endothelial growth factor expression and angiogenesis induced by chronic cerebral hypoperfusion in rat brain. Neurosurgery. 2003;53:963-970; discussion 970-972.

14. Petit I, Szyper-Kravitz M, Nagler A, et al. G-CSF induces stem cell mobilization by decreasing bone marrow SDF-1 and up-regulating CXCR4. Nat Immunol. 2002;3:687-694.

15. Ara T, Tokoyoda K, Okamoto R, Koni PA, Nagasawa T. The role of CXCL12 in the organ-specific process of artery formation. Blood. 2005; 105:3155-3161.

16. Kryczek I, Wei S, Keller E, Liu R, Zou W. Stroma-derived factor (SDF-1/CXCL12) and human tumor pathogenesis. Am J Physiol Cell Physiol. 2007;292:C987-C995.

17. Kollet O, Shivtiel S, Chen YQ, et al. HGF, SDF-1, and MMP-9 are involved in stress-induced human $\mathrm{CD}_{3} 4^{+}$stem cell recruitment to the liver. J Clin Invest. 2003;112:160.

18. Shiba Y, Takahashi M, Yoshioka T, et al. M-CSF accelerates neointimal formation in the early phase after vascular injury in mice the critical role of the SDF-1-CXCR4 system. Arterioscler Thromb Vasc Biol. 2007;27: 283-289.

19. Ponomaryov T, Peled A, Petit I, et al. Induction of the chemokine stromal-derived factor-1 following DNA damage improves human stem cell function. J Clin Invest. 2000;106:1331-1339.

20. Ceradini DJ, Kulkarni AR, Callaghan MJ, et al. Progenitor cell trafficking is regulated by hypoxic gradients through HIF-1 induction of SDF-1. Nat Med. 2004;10:858-864.

21. Salvucci O, Yao L, Villalba S, Sajewicz A, Pittaluga S, Tosato G. Regulation of endothelial cell branching morphogenesis by endogenous chemokine stromal-derived factor-1. Blood. 2002;99:2703-2711.

22. Kryczek I, Lange A, Mottram P, et al. CXCL12 and vascular endothelial growth factor synergistically induce neoangiogenesis in human ovarian cancers. Cancer Res. 2005;65:465-472.

23. Jin DK, Shido K, Kopp HG, et al. Cytokine-mediated deployment of SDF-1 induces revascularization through recruitment of $\mathrm{CXCR}^{+}$ hemangiocytes. Nat Med. 2006;12:557-567.
Therapeutics and Clinical Risk Management

\section{Publish your work in this journal}

Therapeutics and Clinical Risk Management is an international, peerreviewed journal of clinical therapeutics and risk management, focusing on concise rapid reporting of clinical studies in all therapeutic areas, outcomes, safety, and programs for the effective, safe, and sustained use of medicines. This journal is indexed on PubMed Central, CAS,
Dovepress

EMBase, Scopus and the Elsevier Bibliographic databases. The manuscript management system is completely online and includes a very quick and fair peer-review system, which is all easy to use. Visit $\mathrm{http}: / / \mathrm{www}$.dovepress.com/testimonials.php to read real quotes from published authors. 